\title{
Wess-Zumino-Witten and fermion models in noncommutative space
}

\author{
E. Moreno* and F.A. Schaposnik ${ }^{\dagger}$ \\ Departamento de Física, Universidad Nacional de La Plata \\ C.C. 67, 1900 La Plata, Argentina
}

\begin{abstract}
We analyze the connection between Wess-Zumino-Witten and free fermion models in two-dimensional noncommutative space. Starting from the computation of the determinant of the Dirac operator in a gauge field background, we derive the corresponding bosonization recipe studying, as an example, bosonization of the $U(N)$ Thirring model. Concerning the properties of the noncommutative Wess-Zumino-Witten model, we construct an orbit-preserving transformation that maps the standard commutative WZW action into the noncommutative one.
\end{abstract}

\section{Introduction}

Noncommutative field theories have recently attracted much attention in connection with the low-energy dynamics of D-branes in the presence of a background $B$ field [1]-[3]. Afterwards, the unusual properties that emerged

*Investigador CONICET, Argentina.

${ }^{\dagger}$ Investigador CICBA, Argentina 
in the analysis of such field theories made them very attractive by its own right [4]- [8].

Concerning two-dimensional noncommutative field theories, both bosonic and fermionic models have been recently investigated [9]-13. In particular, we have proven in [9] that the fermion determinant of the Dirac operator for fermions in a gauge field background can be written as a Wess-ZuminoWitten (WZW) Lagrangian. As in the ordinary commutative case, this result can be exploited to establish a bosonization recipe for non-commutative fermion models, as developed in [9], [13].

In this paper we pursue the analysis of two-dimensional noncommutative models by carefully studying the fermion effective action. Particular care is taken of the fact that, even in the $U(1)$ case, the noncommutative Dirac operator can be constructed in different representations which can be thought of as the fundamental, anti-fundamental and adjoint ones. As we shall see, this plays an important role in connection with the resulting bosonized theory. As an example, we discuss the $U(1)$ massless Thirring model. We also consider the case in which fermions are taken in the fundamental representation of $U(N)$. Concerning the properties of the bosonic model, we establish a mapping connecting the noncommutative WZW action to the standard one and discuss its relation with the Seiberg-Witten [3] mapping for gauge theories.

The plan of the paper is the following: after establishing our conventions in section 2, we describe in section 3 the evaluation of the perturbative effective action for $U(1)$ noncommutative two-dimensional fermions in a gauge field background. We also discuss the extension of this calculation to the $U(N)$ case. Then, we present the exact evaluation of the fermion determinant both in the fundamental and in the adjoint representations (sections 4 and 5 respectively) In section 6 we present the bosonization recipe which allows to infer a mapping between non-commutative and commutative WZW models (Section 7). In section 8 we apply our results to the analysis of the noncommutative Thirring model and give our conclusions in section 9 . 


\section{Two-dimensional fermion determinants in non-commutative space}

We shall work in two-dimensional Euclidean space and define the $*$ product between functions $\phi(x)$ and $\chi(x)$ in the form

$$
\phi(x) * \chi(x)=\left.\exp \left(\frac{i}{2} \theta_{\mu \nu} \partial_{x}^{\mu} \partial_{y}^{\nu}\right) \phi(x) \chi(y)\right|_{x=y}
$$

where $\theta_{\mu \nu}=\theta \varepsilon_{\mu \nu}$ with $\theta$ a real constant. Then, the Moyal bracket is defined as

$$
\{\phi(x), \chi(x)\}=\phi(x) * \chi(x)-\chi(x) * \phi(x)
$$

which implies a noncommutative relation for space-time coordinates $x^{\mu}$,

$$
\left\{x^{\mu}, x^{\nu}\right\}=i \theta_{\mu \nu}
$$

In the case of gauge theories, noncommutativity leads to the definition of the curvature $F_{\mu \nu}$ in the form

$$
F_{\mu \nu}=\partial_{\mu} A_{\nu}-\partial_{\nu} A_{\mu}-i e\left\{A_{\mu}, A_{\nu}\right\}
$$

Gauge transformations are defined in the form

$$
A_{\mu}^{g}(x)=g(x) * A_{\mu}(x) * g^{-1}(x)+\frac{i}{e} g^{-1}(x) \partial_{\mu} g(x)
$$

where $g(x)$ is represented by a $*$ exponential,

$$
g(x)=\exp _{*}(i \lambda(x)) \equiv 1+i \lambda(x)-\frac{1}{2} \lambda(x) * \lambda(x)+\ldots
$$

with $\lambda=\lambda^{a} t^{a}$ taking values in the Lie algebra of $U(N)$. The covariant derivative $\mathcal{D}_{\mu}[A]$ implementing gauge transformations takes the form

$$
\mathcal{D}_{\mu} \lambda=\partial_{\mu} \lambda-i e\left\{A_{\mu}, \lambda\right\}
$$

Given a fermion field $\psi(x)$, three alternative infinitesimal gauge transformations can be considered [14]

$$
\begin{aligned}
& \delta_{\epsilon} \psi=i \epsilon(x) * \psi(x) \\
& \delta_{\epsilon} \psi=-i \psi(x) * \epsilon(x) \\
& \delta_{\epsilon} \psi=i\{\epsilon(x), \psi(x)\}
\end{aligned}
$$


In this respect, we should refer to fermions in the fundamental $f$ (eq.(8)), 'anti-fundamental' $\bar{f}$ (eq.(9)) and 'adjoint' ad (eq.(10)) representations. The associated covariant derivative are defined accordingly,

$$
\begin{aligned}
D_{\mu}^{f}[A] \psi(x) & =\partial_{\mu} \psi(x)-i e A_{\mu}(x) * \psi(x) \\
D_{\mu}^{\bar{f}}[A] \psi(x) & =\partial_{\mu} \psi(x)+i e \psi(x) A_{\mu}(x) \\
D_{\mu}^{a d}[A] \psi(x) & =\mathcal{D}_{\mu} \psi(x)
\end{aligned}
$$

Using each one of these three covariant derivatives, a gauge invariant Dirac action for fermions can be constructed

$$
S[\bar{\psi}, \psi, A]=\int d^{2} x \bar{\psi} * i \gamma^{\mu} D_{\mu} \psi
$$

The effective action for fermions in a gauge field background is defined as

$$
\exp \left(-S_{e f f}[A]\right)=\int D \bar{\psi} D \psi \exp (-S[\bar{\psi}, \psi, A])
$$

\section{Perturbative effective action}

Let us start by computing the quadratic part of the effective action defined by eq.(15) when the Dirac operator is taken in the adjoint representation, as defined in eq.(13). The interaction term $S_{I}$ of the action $S[\bar{\psi}, \psi, A]$ (eq.(14])) takes the form, in momentum representation,

$$
S_{I}=2 i e \int_{p, q} \bar{\psi}(p) \gamma^{\mu} \psi(q) A_{\mu}(-p-q) \sin (q \wedge p),
$$

where $\int_{p} \equiv \int d^{2} p /(2 \pi)^{2}$.

The quadratic part $\Gamma^{(2)}$ of the effective action is given by the diagram represented in the figure, 


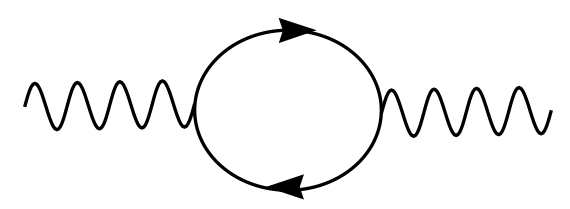

Fig. 1

giving

$$
\Gamma^{(2)}=2 e \int_{p} A_{\mu}(p) A_{\nu}(-p) \int_{q} \sin (q \wedge p)^{2} \operatorname{tr}\left[\frac{(\not q+\not p / 2) \gamma^{\mu}(\not q-\not p / 2) \gamma^{\nu}}{(q+p / 2)^{2}(q-p / 2)^{2}}\right]
$$

Using the identity $\sin (a)^{2}=1 / 2-\cos (2 a) / 2$ we can extract form eq. (17) the so-called planar and non-planar contributions to $\Gamma^{(2)}$,

$$
\Gamma^{(2)}=\int_{p} A_{\mu}(p) A_{\nu}(-p)\left(\Gamma_{\text {planar }}^{\mu \nu(2)}+\Gamma_{\text {nonplanar }}^{\mu \nu(2)}\right)
$$

with

$$
\begin{aligned}
\Gamma_{\text {planar }}^{\mu \nu(2)}=2 e \int_{q} & \left\{\frac{\left(q^{\mu}+p^{\mu} / 2\right)\left(q^{\nu}-p^{\nu} / 2\right)+\left(q^{\mu}-p^{\mu} / 2\right)\left(q^{\nu}+p^{\nu} / 2\right)}{(q+p / 2)^{2}(q-p / 2)^{2}}\right. \\
& \left.-\frac{\delta^{\mu \nu}(q+p / 2) \cdot(q-p / 2)}{(q+p / 2)^{2}(q-p / 2)^{2}}\right\} \\
\Gamma_{\text {nonplanar }}^{\mu \nu(2)}=-2 e \int_{q}\left\{\frac{\left(q^{\mu}+p^{\mu} / 2\right)\left(q^{\nu}-p^{\nu} / 2\right)+\left(q^{\mu}-p^{\mu} / 2\right)\left(q^{\nu}+p^{\nu} / 2\right)}{(q+p / 2)^{2}(q-p / 2)^{2}}\right. & \left.-\frac{\delta^{\mu \nu}(q+p / 2) \cdot(q-p / 2)}{(q+p / 2)^{2}(q-p / 2)^{2}}\right\} \cos (2 q \wedge p)
\end{aligned}
$$

The planar contribution to the diagram is the standard one and can be computed using for example dimensional regularization (in this case the 
infrared an ultraviolet divergences cancel each other). One has

$$
\Gamma_{\text {planar }}^{\mu \nu(2)}=\frac{e}{\pi}\left(\delta^{\mu \nu}-\frac{p^{\mu} p^{\nu}}{p^{2}}\right)
$$

It is worthwhile to mention that this result is twice the effective action in the fundamental and anti-fundamental representations (that is, taking the Dirac operator as defined either by (11) or by (12)), as it can be easily seen by noticing that in the later the diagram has a vertex contribution of $e^{i p \wedge q} e^{-i p \wedge q}=1$ (there is no non-planar contribution) while in the former we have $-\left(2 i \sin (p \wedge q)^{2}=4(1 / 2-1 / 2 \cos (2 p \wedge q))\right.$. Taking into account that the only the non-oscillating part contributes to the planar part of the diagram we have

$$
\Gamma_{\text {planar }}^{(2) A d j}=2 \Gamma^{(2) \text { Fund }}
$$

Interestingly enough, this is reminiscent of the relation that one obtains when one compares the anomaly and the fermion determinant for commutative two-dimensional fermions in an $U(N)$ gauge field background, for the fundamental and the adjoint representation of $U(N)$. In this case, there is a factor relating the results in the adjoint and the fundamental which corresponds to the quadratic Casimir $C(G)$ in the adjoint, as first reported in [17] (see for example [18] for a detailed derivation). Now, it was observed in [19] 21] that diagrams in noncommutative $U(1)$ gauge theories could be constructed in terms of those in ordinary non-Abelian gauge theory with $C(G)=2$; this is precisely what we have found in the present 2-dimensional model. Note that the explicit form of integrals associated to the diagram in Fig.1 for each fermion representation can be constructed if one take as generators for the Moyal algebra generators $t$ and $T$ in the adjoint and the fundamental representation such that

$$
\begin{aligned}
& t_{p k}^{q}=2 i \sin (k \wedge p) \delta(k+p-q) \\
& T_{p k}^{q}=\exp (i k \wedge p) \delta(k+p-q)
\end{aligned}
$$

Let us now compute the non-planar contribution to $\Gamma^{(2)}$. First we exponentiate the propagators with Schwinger parameters

$$
\frac{1}{(q+p / 2)^{2}(q-p / 2)^{2}}=\int_{0}^{\infty} d t_{1} \int_{0}^{\infty} d t_{2} e^{-t_{1}(q+p / 2)^{2}-t_{2}(q-p / 2)^{2}} .
$$


Afterwards we can perform the $q$ integration, which becomes gaussian, and we get (after the standard redefinition $t_{1}=u s$ and $\left.t_{2}=(1-u) s\right)$ ) :

$$
\begin{aligned}
\Gamma_{\text {non-planar }}^{\mu \nu(2)} & =-\frac{e}{2 \pi} \int_{0}^{\infty} d s \int_{0}^{1} d u\left\{\delta^{\mu \nu}\left(\frac{1}{s}+u(1-u) p^{2}+\frac{1}{4 s}(\theta \cdot p)^{2}\right)\right. \\
- & \left.2 p^{\mu} p^{\nu} u(1-u)-\frac{1}{2 s}(\theta \cdot p)^{\mu}(\theta \cdot p)^{\nu}\right\} e^{-s u(1-u) p^{2}-\frac{1}{4 s}(\theta \cdot p)^{2}},
\end{aligned}
$$

where $(\theta \cdot p)^{\mu}=\theta^{\mu \nu} p_{\nu}$. Finally the $s$ integration can also be performed and we obtain the expression:

$$
\Gamma_{n o n-p l a n a r}^{\mu \nu(2)}=-\frac{1}{2 \pi}\left\{\left(\delta^{\mu \nu}-\frac{p^{\mu} p^{\nu}}{p^{2}}\right)-\frac{(\theta \cdot p)^{\mu}(\theta \cdot p)^{\nu}}{(\theta \cdot p)}\right\} \int_{0}^{1} d u x K_{1}(x)
$$

where $x^{2}=u(1-u) p^{2}(\theta \cdot p)^{2}$ and $K_{1}(x)$ is the modified Bessel function of second kind. Notice that the $u$ integral converges.

Now, in two dimensions $\theta^{\mu \nu}=\theta \epsilon^{\mu \nu}$ so we have the identity:

$$
\frac{(\theta \cdot p)^{\mu}(\theta \cdot p)^{\nu}}{(\theta \cdot p)^{2}}=\delta^{\mu \nu}-\frac{p^{\mu} p^{\nu}}{p^{2}}
$$

and thus $\Gamma_{n o n-p l a n a r}^{(2)}$ vanishes. That is, up to quadratic order in the fields, the effective action is given by the planar part:

$$
\Gamma^{(2)}=\frac{e}{\pi} \int_{p} A_{\mu}(p)\left(\delta^{\mu \nu}-\frac{p^{\mu} p^{\nu}}{p^{2}}\right) A_{\nu}(-p) .
$$

Therefore, assuming that the higher point contributions to the effective action are the minimal necessary to recover gauge invariance (we will prove this statement in the next section), the effective action in the adjoint representation is twice the effective action in the fundamental representation. Hence, a relation like (22) should hold for the complete effective action $\Gamma$

$$
\Gamma^{A d j}=2 \Gamma^{\text {Fund }}
$$

This computation can be generalized to the $U(N)$ case, namely when the Dirac operator acts as

$$
\mathcal{D}_{\mu} \lambda=\partial_{\mu} \lambda-i e\left\{A_{\mu}, \lambda\right\}
$$


with $\lambda \equiv \lambda^{a} t^{a}$. In this case the interaction part of the action takes the form $S_{I}=e \sqrt{2} \int_{p, q} \bar{\psi}^{a}(p) \gamma^{\mu} \psi(q)^{b} A_{\mu}^{c}(-p-q)\left(i d^{a b c} \sin (q \wedge p)+i f^{a b c} \cos (q \wedge p)\right)$

where the $f^{a b c}$ and $d^{a b c}$ are, respectively, the antisymmetric and symmetric structure constants of $U(N)$ (the $\sqrt{2} e$ factor comes from our normalization of the $U(N)$ generators).

The quadratic part of the effective action is in this case

$$
\begin{array}{r}
\Gamma^{(2)}=\frac{e}{2} \int_{p} A_{\mu}^{a}(p) A_{\nu}^{b}(-p) \int_{q} \operatorname{tr}\left[\frac{(\not 1+\not p / 2) \gamma^{\mu}(\not 1-\not p / 2) \gamma^{\nu}}{(q+p / 2)^{2}(q-p / 2)^{2}}\right] \times \\
\left\{\left(d^{a c d} d^{b c d}+f^{a c d} f^{b c d}\right)+\left(d^{a c d} d^{b c d}-f^{a c d} f^{b c d}\right) \cos (2 q \wedge p)\right\}
\end{array}
$$

The planar and non-planar contributions are the same as in the $U(1)$ case except for the group theoretical prefactors. Taking into account that the non-planar contribution vanishes and the identity:

$$
d^{a c d} d^{b c d}+f^{a c d} f^{b c d}=2 N \delta^{a b}
$$

we finally have

$$
\Gamma^{(2)}=N \frac{e}{\pi} \int_{p} A_{\mu}^{a}(p)\left(\delta^{\mu \nu}-\frac{p^{\mu} p^{\nu}}{p^{2}}\right) A_{\nu}^{a}(-p) .
$$

This result is $2 N$ times the quadratic effective action in the fundamental representation.

\section{The fermion determinant in the fundamen- tal representation: the $U(1)$ case}

Here we shall briefly describe the exact calculation of the effective action for noncommutative $U(1)$ fermions in the fundamental representation as first presented in ref. 99 by integrating the chiral anomaly. Indeed, taking profit that in 2 dimensions a gauge field $A_{\mu}$ can always be written in the form

$$
\not A=-\frac{1}{e}(i \not \partial U[\phi, \eta]) * U^{-1}[\phi, \eta]
$$


with

$$
U[\phi, \eta]=\exp _{*}\left(\gamma_{5} \phi+i \eta\right),
$$

one can relate the fermion determinant in a gauge field background $A_{\mu}$ with that corresponding to $A_{\mu}=0$ by making a decoupling change of variables in the fermion fields. As announced, we consider in this section the case of fermions in the fundamental representation. Then, the appropriate change of fermionic variables is

$$
\psi \rightarrow U[\phi, \eta] * \psi, \quad \bar{\psi} \rightarrow \bar{\psi} * U^{-1}[\phi, \eta]
$$

One gets [9]

$$
\operatorname{det}(\not \partial-i e \not A)=\operatorname{det} \not \partial \exp \left(-2 \int_{0}^{1} d t \frac{d J^{f}[t \phi, A]}{d t}\right)
$$

where $J^{f}[t \phi, A]$ is the Fujikawa Jacobian associated with a transformation $U_{t}$ such that $(o \leq t \leq 1)$

$$
\begin{aligned}
& U_{0}=1 \\
& U_{1}=U[\phi, \eta]
\end{aligned}
$$

Let us briefly describe at this point the calculation presented in [9] based in the the evaluation of the chiral anomaly. Consider an infinitesimal local chiral transformation which in the fundamental representation reads

$$
\delta_{\epsilon}^{5} \psi=i \gamma_{5} \epsilon(x) * \psi(x)
$$

The chiral anomaly $\mathcal{A}=\mathcal{A}^{a} t^{a}$,

$$
\begin{gathered}
\partial_{\mu} j_{5}^{a \mu}=\mathcal{A}^{a}[A], \\
j_{5}^{a \mu}=\bar{\psi} \gamma_{5} t^{a} \psi
\end{gathered}
$$

can be calculated from the Fujikawa Jacobian $J\left[\epsilon, A_{\mu}\right]$ associated with infinitesimal transformation (41),

$$
\log J\left[\epsilon, A_{\mu}\right]=-2 \operatorname{tr}^{\mathrm{c}} \int d^{2} x \mathcal{A}[A] \epsilon(x)=-\left.2 \operatorname{tr}^{\mathrm{c}} \int d^{2} x \operatorname{Tr}\left(\gamma_{5} \epsilon(x)\right)\right|_{\text {reg }}
$$


Here Tr means both a trace for Dirac (tr) and a functional trace in the space on which the Dirac operator acts while $\operatorname{tr}^{c}$ indicates a trace over the gauge group indices. We indicate with reg that some regularization prescription should be adopted to render finite this trace. We shall adopt the heat-kernel regularization, this meaning that

$$
\mathcal{A}[A]=\lim _{M^{2} \rightarrow \infty} \operatorname{Tr}\left(\gamma_{5} \exp _{*}\left(\frac{\gamma^{\mu} \gamma^{\nu} D_{\mu} * D_{\nu}}{M^{2}}\right)\right)
$$

The covariant derivative in the regulator has to be chosen among those defined by eqs.(11)-(13) according to the representation one has chosen for the fermions. Concerning the fundamental representation, the anomaly has been computed following the standard Fujikawa procedure [15] in [16], [9].

$$
\mathcal{A}^{f}[A]=\frac{e}{4 \pi} \varepsilon^{\mu \nu} F_{\mu \nu}
$$

(We indicate with $f$ that the fundamental representation has been considered). Analogously, one obtains for the anti-fundamental representation

$$
\mathcal{A}^{\bar{f}}[A]=-\frac{e}{4 \pi} \varepsilon^{\mu \nu} F_{\mu \nu}
$$

Now, writing $\epsilon=\phi d t$, we can use the results given through eqs.(44)-(46) to get, for the Jacobian,

$$
\log J^{f}[t \phi, A]=-\frac{e}{2 \pi} \operatorname{tr}^{\mathrm{c}} \int d^{2} x \varepsilon_{\mu \nu} F_{\mu \nu}^{t} * \phi
$$

where

$$
\begin{gathered}
F_{\mu \nu}^{t}=\partial_{\mu} A_{\nu}-\partial_{\nu} A_{\mu}-i e\left\{A_{\mu}^{t}, A_{\nu}^{t}\right\} \\
\gamma^{\mu} A_{\mu}^{t}=-\frac{1}{e}\left(i \not \partial U_{t}\right) * U_{t}^{-1}
\end{gathered}
$$

This result can be put in a more suggestive way in the light cone gauge where

$$
\begin{aligned}
A_{+} & =0 \\
A_{-} & =g(x) * \partial_{-} g^{-1}(x) \\
g(x) & =\exp _{*}(2 \phi)
\end{aligned}
$$


Indeed, in this gauge one can see that (48) becomes

$$
\begin{gathered}
\log \left(\frac{\operatorname{det}(\not \partial-i e A)}{\operatorname{det} \not \partial}\right)=\log J^{f}[t \phi, A]=-\frac{1}{8 \pi} \operatorname{tr}^{\mathrm{c}} \int d^{2} x\left(\partial_{\mu} g^{-1}\right) *\left(\partial_{\mu} g\right) \\
+\frac{i}{12 \pi} \varepsilon_{i j k} \operatorname{tr}^{\mathrm{c}} \int_{B} d^{3} y g^{-1} *\left(\partial_{i} g\right) * g^{-1} *\left(\partial_{j} g\right) * g^{-1} *\left(\partial_{k} g\right)
\end{gathered}
$$

Here we have written $d^{3} y=d^{2} x d t$ so that the integral in the second line runs over a 3-dimensional manifold $B$ which in compactified Euclidean space can be identified with a ball with boundary $S^{2}$. Index $i$ runs from 1 to 3 . Concerning the anti-fundamental representation, the calculation of the fermion determinant follows identical steps. Using the expression of the anomaly given by (47), one computes the determinant which coincides with that in the fundamental representation (remember that the anomaly is proportional to the charge $e$ while the determinant to $\left.e^{2}\right)$. These were the main results in ref.[9]. In the next section, we shall extend them to the case in which the Dirac operator is in the adjoint representation.

\section{The determinant in the adjoint representa- tion: the $U(1)$ case}

We showed in Section 3 that the effective action for the Dirac fermions coupled to an external gauge field in the adjoint representation is, at quadratic order, twice the effective action in the fundamental representation. In this section we will find the exact effective action by computing in exact form the fermionic determinant with the heat-kernel approach.

The action is given by

$$
S_{f}=\int d^{2} x \bar{\psi} * i \gamma^{\mu}\left(\partial_{\mu} \psi+i e\left\{A_{\mu}, \psi\right\}\right)
$$

Writing the field $A_{\mu}$ as in equation (36) (for simplicity we ill work in the gauge $\eta=0$ ) we can make a change if the fermion variables to decouple the fermions from the gauge field

$$
\begin{aligned}
& \psi=e^{\gamma^{5}\{\phi, \cdot\}} * \chi=\chi+\gamma^{5}\{\phi, \chi\}+\frac{1}{2}\{\phi,\{\phi, \chi\}\}+\cdots \\
& \bar{\psi}=\bar{\chi} * e^{\{\cdot, \phi\} \gamma^{5}}=\bar{\chi}+\{\bar{\chi}, \phi\} \gamma^{5}+\frac{1}{2}\{\{\bar{\chi}, \phi\}, \phi\}+\cdots
\end{aligned}
$$


Similarly to the previous section, the Fujikawa Jacobian associated to (54) can bee written as

$$
\operatorname{det}\left(\not \partial+i e \gamma \cdot[A, \cdot]_{*}\right)=\operatorname{det} \not \partial \exp \left(-2 \int_{0}^{!} d t \frac{d J^{a d}[t \phi, A]}{d t}\right)
$$

where $J^{a d}[t \phi, A]$ is the Fujikawa Jacobian associated with a transformation for fermions in the adjoint,

$$
\begin{aligned}
& \psi=e^{\gamma^{5}[t \phi,]_{*}} * \chi \\
& \bar{\psi}=\bar{\chi} * e^{[\cdot, t \phi]_{*} \gamma^{5}}
\end{aligned}
$$

where $t$ is a parameter.

Following the same procedure as before, we find that

$$
\frac{d}{d t} \log \left(J^{a d}\right)=\int d^{2} x \mathcal{A}(x) * \phi(x)
$$

where

$$
\mathcal{A}(x)=2 \lim _{M \rightarrow \infty} \operatorname{tr} \gamma^{5} \int_{k} \exp _{*}(-i k \cdot x) \exp _{*}\left(\not D^{2} A d / M^{2}\right) \exp _{*}(i k \cdot x)
$$

After a straightforward computation it can be proved the following identity

$$
\exp _{*}(-i k \cdot x) \exp _{*}\left(\not D_{A d}^{2} / M^{2}\right) \exp _{*}(i k \cdot x)=\exp _{*}\left(\left(\not D_{A d}+i \not k+i e \not\right)^{2} / M^{2}\right)
$$

where

$$
\begin{aligned}
c_{\mu}(x ; k) & =-2 i \int_{p} \exp _{*}(-i p \wedge k \sin (p \wedge k)) A_{\mu}(p) \exp _{*}(i p \cdot x) \\
& =A_{\mu}(x-\theta \cdot k)-A_{\mu}(k)
\end{aligned}
$$

Finally, expanding the exponent up to order $M^{-2}$, taking the trace and integrating over $d^{2} k$ we have

$$
\mathcal{A}(x)=-\frac{e}{\pi} \lim _{M \rightarrow \infty} \int_{p}\left(\left(1-\exp _{*}\left(-M^{2} \theta^{2} p^{2} / 4\right)\right) \epsilon_{\mu \nu} F_{\mu \nu}(p) \exp _{*}(i p \cdot x)\right.
$$

Notice that if we take the $\theta \rightarrow 0$ limit before taking the $M \rightarrow \infty$ limit $\mathcal{A}$ vanishes and the Jacobian is 1 , so we recover the standard (commutative) result which corresponds to a trivial determinant for the trivial $U(1)$ covariant 
derivative. However, the limits $\theta \rightarrow 0$ and $M \rightarrow \infty$ do not commute so that if one takes the $M^{2} \rightarrow \infty$ limit at fixed $\theta$ one has the $\theta$-independent result

$$
\frac{d}{d t} \log J^{A d}[t \phi, A]=-\frac{e}{\pi} \int d^{2} x \varepsilon_{\mu \nu} F_{\mu \nu}^{t} * \phi
$$

which is twice the result of the fundamental representation. The integral in $t$ is identical to the one of the fundamental representation so we finally have

$$
\begin{array}{r}
\log \left(\frac{\operatorname{det}\left(\not \partial-i e \gamma_{\mu}\left\{A_{\mu},\right\}\right)}{\operatorname{det} \not \partial}\right)=-\frac{1}{4 \pi} \operatorname{tr}^{\mathrm{c}} \int d^{2} x\left(\partial_{\mu} g^{-1}\right) *\left(\partial_{\mu} g\right) \\
+\frac{i}{6 \pi} \varepsilon_{i j k} \operatorname{tr}^{\mathrm{c}} \int_{B} d^{3} y g^{-1} *\left(\partial_{i} g\right) * g^{-1} *\left(\partial_{j} g\right) * g^{-1} *\left(\partial_{k} g\right)
\end{array}
$$

We have thus reobtained relation (30) but now by comparing the exact answer for the fermion determinant in both representations.

\section{The bosonization recipe}

Once one has gotten an exact result for the fermion determinant, one can derive the path-integral bosonization recipe. That is, a mapping from the a two-dimensional noncommutative fermionic model with onto an equivalent noncommutative bosonic model. This implies a precise relation between the fermionic and bosonic Lagrangian, currents, etc. The basic procedure to obtain this bosonization recipe parallels that already established in the ordinary commutative case. For the noncommutative case, it was developed in detail in [13]. Here, we shall describe just the main steps in the derivation of this equivalence.

The fermion current associated with the $U(1)$ gauge invariance of the fermion model is naturally given by

$$
j_{\mu}(x)=\bar{\psi}(x) * \gamma_{\mu} \psi(x)
$$

Its coupling to an external source in order to obtain correlation functions after differentiation can be done simply in the form

$$
S_{\text {int }}=\int d^{2} x j_{\mu}(x) s_{\mu}(x)
$$


so that after differentiation with respect to $s$ one gets, as usual, v.e.v.'s of current correlation functions. Now, being the integral in (65) quadratic, one can safely replace the ordinary product between $j$ and $s$ by a $*$ product,

$$
S_{\text {int }}=\int d^{2} x j_{\mu}(x) * s_{\mu}(x)
$$

One can then write the fermion generating functional in the presence of a source $s_{\mu}$ in the form,

$$
Z_{f e r}[s]=\int D \bar{\psi} D \psi \exp \left(-\int d^{2} x \bar{\psi} D_{\mu}^{\bar{f}}[s] \psi\right)=\operatorname{det}\left(i D_{\mu}^{\bar{f}}[s]\right)
$$

The occurrence of the Dirac operator in the antifundamental representation is consistent with definition (66) but one can of course also define the generating functional in terms of $D_{\mu}^{f}$.

Since the fermion determinant in the r.h.s. of eq.(67) is gauge invariant, we can write

$$
Z_{f e r}[s]=Z_{f e r}\left[s^{U}\right]
$$

where, as before, we indicate with $s^{U}$ the gauge transformed of $s$ with a gauge group element $U$ as in eq.(5). One now integrates over $U$ both sides in (68) getting

$$
Z_{\text {fer }}[U]=\mathcal{N} \int D U \operatorname{det}\left(i D_{\mu}^{\bar{f}}\left[s^{U}\right]\right)
$$

At this point, one introduces an auxiliary field $b_{\mu}$ through the identity

$$
s_{\mu}^{U}=b_{\mu}
$$

which in the path-integral $(69)$ can be implemented in the form

$$
Z_{f e r}[U]=\int D b_{\mu} \Delta \delta\left(b_{+}-s_{+}\right) \delta\left(\varepsilon_{\mu \nu} F_{\mu \nu}[b]-\varepsilon_{\mu \nu} F_{\mu \nu}[s]\right) \operatorname{det}\left(i D_{\mu}^{\bar{f}}[b]\right)
$$

Here $\Delta$ can be seen as the Faddeev-Popov determinant associated with the delta function "fixing the gauge" $b_{+}=s_{+}$. The other delta completes the identification (70) (These kind of representations are discussed in detail in [22]). One now introduces a Lagrange multiplier $a$ in order to represent the curvature's delta function. Moreover, one writes

$$
\begin{aligned}
& s_{+}=i \tilde{s}^{-1} * \partial_{+} \tilde{s} \\
& s_{-}=i s * \partial_{-} s^{-1} \\
& b_{+}=i s * b \partial_{-}(s * b)^{-1}
\end{aligned}
$$


One also trades the Lie algebra valued scalar Lagrange multiplier $a$ by a gauge group valued $\hat{a}$ through an analogous relation and then, after repeated use of the Polyakov-Wiegmann identity ends with

$$
Z_{f e r}[s]=\int D \hat{a} D b \exp ((1+2) W[a * \tilde{s} * s * b]) \exp (W[\tilde{s} * s]-W[\hat{a} * \tilde{s} * s])
$$

We have explicitly written the factor $(1+2)$ in front of the first WZW Lagrangian to stress that this term arises not only from the fermion determinant but also from the Jacobian arising in passing from the $b_{\mu}$ integration to the $b$ integration, a Jacobian which corresponds to the determinant of the Dirac operator in the adjoint.

Use of the Polyakov-Wiegman identity allows to factorize the integral over $b$ (that is, the $b$ field completely decouples and can be integrated out). After some manipulations, one finally ends with

$$
Z_{f e r}[s]=\int D a \exp \left(-W[\hat{a}]+\frac{i}{4 \pi} \int d^{2} x\left(s_{+} * \hat{a} * \partial_{-} \hat{a}^{-1}+s_{-} * \hat{a}^{-1} * \partial_{+} \hat{a}\right)\right)
$$

(We have ignored terms quadratic in the source which just give irrelevant contact terms when computing current-current correlators).

We have then arrived to the identity

$$
Z_{f e r}[s]=Z_{b o s}[s]
$$

where $Z_{b o s}$ in the r.h.s. is the generating functional for a Wess-ZuminoWitten model for a gauge-group valued field $a$ coupled to bosonic sources in such a way that one can already give the bosonization recipe for currents

$$
\begin{aligned}
& \bar{\psi} * \gamma_{+} \psi \longrightarrow \frac{i}{4 \pi} \hat{a}^{-1} * \partial_{+} \hat{a} \\
& \bar{\psi} * \gamma_{-} \psi \longrightarrow \frac{i}{4 \pi} \hat{a} * \partial_{-} \hat{a}^{-1}
\end{aligned}
$$

We have then proved the equivalence between a two-dimensional noncommutative free fermion model and the non-commutative Wess-Zumino-Witten model. Now, on the one hand we know that, being quadratic, the action for noncommutative free fermions coincides with that for ordinary (commutative) ones. On the other hand we know that ordinary free fermions are 
equivalent to a bosonic theory with ordinary WZW term. The situation can be represented in the following figure

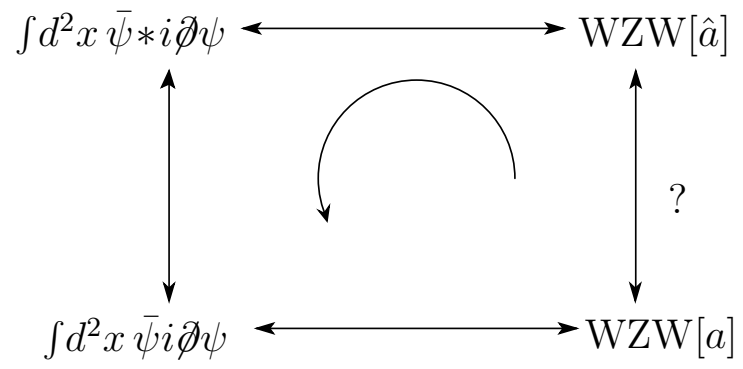

Fig. 2

So, we were able to pass from the noncommutative WZW theory with action $W Z W[\hat{a}]$ to the commutative one, $W Z W[a]$ by going counterclockwise through the fermionic equivalent models. One should then be able to find a mapping $\hat{a} \rightarrow a$, analogous to the one introduced in [3] for non-commutative gauge theories. In the present case, the mapping should connect exactly $W Z W[\hat{a}]$ with $W Z W[a]$ filling the cycle in figure 2 . Next section is devoted to the construction of such a mapping.

\section{Mapping Wess-Zumino-Witten actions: Seiberg-Witten change of variables}

Before study the mapping between the non-commutative and standard WZW theories, let us mention some properties of the Moyal deformation in two dimensions.

Equation (四) can be re-written in term of holomorphic and anti-holomorphic coordinates in the form:

$$
\phi(z, \bar{z}) * \chi(z, \bar{z})=\left.\exp \left\{\theta\left(\partial_{z} \partial_{\bar{w}}-\partial_{\bar{z}} \partial_{w}\right)\right\} \phi(z, \bar{z}) \chi(w, \bar{w})\right|_{w=z}
$$

This equation reduces considerably in two particular cases. First, when one of the functions is holomorphic (anti-holomorphic) and the other is antiholomorphic (holomorphic), the deformed product reads as

$$
\phi(z) * \chi(\bar{z})=e^{\theta \partial_{z} \partial_{\bar{z}}} \phi(z) \chi(\bar{z}), \quad \phi(\bar{z}) * \chi(z)=e^{-\theta \partial_{z} \partial_{\bar{z}}} \phi(\bar{z}) \chi(z)
$$


and the deformation is produced by an overall operation over the standard (commutative) product with no $w \rightarrow z$ limit necessary.

Second, if both functions are holomorphic (anti-holomorphic), the star product coincides with the regular product

$$
\phi(z) * \chi(z)=\phi(z) \chi(z), \quad \phi(\bar{z}) * \chi(\bar{z})=\phi(\bar{z}) \chi(\bar{z}) .
$$

That means that the holomorphic or anti-holomorphic sectors of a twodimensional field theory are unchanged by the deformation of the product. For example, the holomorphic fermionic current in the deformed theory takes the form,

$$
\hat{\mathrm{j}}_{z}=\psi_{R}^{\dagger} * \psi_{R} \text {. }
$$

And since $\psi_{R}$ has no $\bar{z}$ dependence on-shell, the deformed current coincides with the standard one

$$
j_{z}=\psi_{R}^{\dagger} \psi_{R}
$$

Moreover, since the free actions are identical, any correlation functions of currents in the standard and the $*$-deformed theory will be identical.

This last discussion tell us that, since the WZW actions are the generating actions of fermionic current correlation functions, both actions (standard and non-commutative) are equivalent. It remains to see if we can link both actions through a Seiberg-Witten like mapping. Let us try that.

Consider a WZW action defined in a non-commutative space with deformation parameter $\theta$. The action is invariant under chiral holomorphic and anti-holomorphic transformations

$$
\hat{g} \rightarrow \bar{\Omega}(\bar{z}) \hat{g} \Omega(z)
$$

so in analogy with the Seiberg-Witten mapping we will look for a transformation that maps respectively holomorphic and anti-holomorphic "orbits" into "orbits". Of course the analogy breaks down at some point as this holomorphic and anti-holomorphic "orbits" are not equivalence classes of physical configurations, but just symmetries of the action. However we will see that such a requirement is equivalent, in some sense, to the "gauge orbits preserving transformation condition" of Seiberg-Witten.

Thus, we will find a transformation that maps a group-valued field $\hat{g}^{\prime}$ defined in non-commutative space with deformation parameter $\theta^{\prime}$ to a group- 
valued field $\hat{g}$, with deformation parameter $\theta$. We demand this transformation to satisfy the condition

$$
\bar{\Omega}^{\prime}(\bar{z}) *^{\prime} \hat{g}^{\prime} *^{\prime} \Omega^{\prime}(z) \rightarrow \bar{\Omega}(\bar{z}) * \hat{g} * \Omega(z)
$$

where the primed quantities are defined in a $\theta^{\prime}$-non-commutative space and the non primed quantities defined in a $\theta$-non-commutative space. In particular this mapping will preserve the equations of motion:

$$
\hat{g}^{\prime}=\alpha^{\prime} *^{\prime} \beta^{\prime} \rightarrow \hat{g}=\alpha * \beta
$$

The simplest way to achieve this, by examining equation (78) is defining

$$
\hat{g}[\theta]=e^{-\theta \partial_{z} \partial_{\bar{z}}} g[0]
$$

or, infinitesimally

$$
\frac{d \hat{g}}{d \theta}=-\partial_{z} \partial_{\bar{z}} \hat{g}
$$

However, the corresponding transformation for $\hat{g}^{-1}$ is more cumbersome

$$
\frac{d \hat{g}^{-1}}{d \theta}=\partial_{z} \partial_{\bar{z}} \hat{g}^{-1}+2 \partial_{\bar{z}}\left(\hat{g}^{-1} * \partial_{z} \hat{g}\right) * \hat{g}^{-1}
$$

So let us consider a more symmetric transformation, that coincides on-shell, with (86) and (87). Consider thus

$$
\begin{aligned}
\frac{d \hat{g}}{d \theta} & =\hat{g} * \partial_{\bar{z}} \hat{g}^{-1} * \partial_{z} \hat{g} \\
\frac{d \hat{g}^{-1}}{d \theta} & =-\partial_{z} \hat{g}^{-1} * \partial_{\bar{z}} \hat{g} * \hat{g}^{-1}
\end{aligned}
$$

These equations satisfy the condition (83) for functions $\Omega(z)$ and $\bar{\Omega}(\bar{z})$ independent of $\theta$. Indeed we have, for example

$$
\begin{aligned}
\frac{d(\hat{g} * \Omega(z))}{d \theta} & =\frac{d \hat{g}}{d \theta} * \Omega(z)-\partial_{\bar{z}} \hat{g} * \partial_{z} \Omega(z) \\
& =(\hat{g} * \Omega(z)) * \partial_{\bar{z}}(\hat{g} * \Omega(z))^{-1} * \partial_{z}(\hat{g} * \Omega(z))
\end{aligned}
$$

and a similar equation for the anti-holomorphic transformation. 
The next step is to see how does the WZW action transforms under this mapping. First consider the variation of the following object:

$$
\omega=\hat{g}^{-1} * \delta \hat{g}
$$

where $\delta$ is any variation that does not acts on $\theta$.

After a straightforward computation we find that

$$
\frac{d \omega}{d \theta}=-\partial_{\bar{z}} \omega * j_{z}-j_{z} * \partial_{\bar{z}} \omega
$$

where

$$
j=\hat{g}^{-1} * \partial_{z} \hat{g}
$$

is the holomorphic current. In particular we have

$$
\frac{d j_{z}}{d \theta}=-\partial_{\bar{z}} j_{z} * j_{z}-j_{z} * \partial_{\bar{z}} j_{z}=-\partial_{\bar{z}}\left(j_{z}^{2}\right)
$$

Similarly we can find the variations for

$$
\bar{\omega}=\delta \hat{g} * \hat{g}^{-1}
$$

and we get

$$
\begin{aligned}
& \frac{d \bar{\omega}}{d \theta}=\partial_{z} \omega * j_{\bar{z}}+j_{\bar{z}} * \partial_{z} \bar{\omega} \\
& \frac{d j_{\bar{z}}}{d \theta}=\partial_{z} j_{\bar{z}} * j_{\bar{z}}+j_{\bar{z}} * \partial_{z} j_{\bar{z}}=\partial_{z}\left(j_{\bar{z}}^{2}\right)
\end{aligned}
$$

where

$$
j_{\bar{z}}=\partial_{\bar{z}} \hat{g} * \hat{g}^{-1} \text {. }
$$

Note that on-shell, both $j_{z}$ and $j_{\bar{z}}$ are $\theta$-independent, that is the non-commutative currents coincide with the standard ones. This result is expected since the same happens for their fermionic counterparts.

Now, instead of studying how does the $\theta$-map acts on the WZW action, it is easy to see how does the mapping acts on the variation of the WZW action with respect to the fields. In fact, we have

$$
\delta W[\hat{g}]=\frac{k}{\pi} \int d^{2} x \operatorname{tr}\left(\partial_{\bar{z}} j_{z} \omega\right)
$$


where $j_{z}$ and $\omega$ are the quantities defines in eqs.(90) and (92) and there is no *-product between them in eq.(97) in virtue of the quadratic nature of the expression.

Thus, a simple computation show that

$$
\frac{d \delta W[\hat{g}]}{d \theta}=0
$$

and we have a remarkable result: the transformation (88), integrated between 0 and $\theta$ maps the standard commutative WZW action into the noncommutative WZW action. That is, we have found a transformation mapping orbits into orbits such that it keeps the form of the action unchanged provided one simply performs a $\theta$-deformation. This should be contrasted with the 4 dimensional noncommutative Yang-Mills case for which a mapping respecting gauge orbits can be found (the Seiberg-Witten mapping) but the resulting commutative action is not the standard Yang-Mills one. However, one can see that the mapping (88) is in fact a kind of Seiberg-Witten change of variables.

Indeed, if we consider the WZW action as the effective action of a theory of Dirac fermions coupled to gauge fields, as we did in previous sections, instead of an independent model, we can relate the group valued field $g$ to gauge potentials. As we showed in eq.(51), this relation acquires a very simple form in the light-cone gauge $A_{+}=0$ where

$$
A_{-}=\hat{g}(x) * \partial_{-} \hat{g}^{-1}(x) .
$$

But notice that in this gauge, $A_{-}$coincides with $j_{\bar{z}}$ (eq.(96), so we have from equation (95)

$$
\delta A_{-}=\delta \theta\left(\partial_{z} A_{-} * A_{-}+A_{-} * \partial_{z} A_{-}\right)
$$

which is precisely the Seiberg-Witten mapping in the gauge $A_{+}=0$.

\section{The $U(1)$ Thirring model}

As an example of the bosonization recipe previously obtained, we analyze in this section the two-dimensional non-commutative Thirring model with dynamics governed by the (Euclidean) Lagrangian

$$
L=\bar{\psi} * i \not \partial \psi-\frac{g^{2}}{2} j_{\mu} * j^{\mu}
$$


where the fermion current is defined as

$$
j_{\mu}=\bar{\psi} * \gamma_{\mu} \psi
$$

The partition function for the model is

$$
Z=\int D \bar{\psi} D \psi \exp \left(-\int d^{2} x L\right)
$$

Let us introduce an auxiliary vector field $A_{\mu}$ to eliminate the quartic fermion self-interaction. We use the identity

$$
\exp \left(\frac{g^{2}}{2} \int d^{2} x j_{\mu} * j^{\mu}\right)=\int D A_{\mu} \exp \left(-\int d^{2} x\left(\frac{1}{2 g^{2}} A_{\mu} * A^{\mu}-j_{\mu} * A_{\mu}\right)\right)
$$

Being all terms in the exponentials quadratic, the $*$ product in (104) can be replaced by the normal one so that (104) is just the usual HubbardStratonovich identity.

With this, the partition function (103) can be written as

$$
Z=\int D \bar{\psi} D \psi D A_{\mu} \exp \left(-\int d^{2} x L_{e f f}\right)
$$

where we have defined

$$
L_{e f f}=\bar{\psi} * \gamma^{\mu} i D_{\mu}^{\bar{f}}[A] \psi+\frac{1}{2 g^{2}} A_{\mu} * A^{\mu}
$$

Here the Dirac operator $D_{\mu}^{\bar{f}}[A]$ acts on the anti-fundamental representation (as defined in eq.(12) with $e=1$ ). The fermionic path-integral in $Z$ can now be performed, leading to

$$
Z=\int D A_{\mu} \exp \left(-\frac{1}{2 g^{2}} \int d^{2} x A_{\mu} * A^{\mu}\right) \operatorname{det}\left(\gamma^{\mu} i D_{\mu}^{\bar{f}}[A]\right)
$$

In order to use the results obtained in the previous sections, we write, for the vector field light-cone components,

$$
\begin{aligned}
& A_{+}=g^{-1}(x) * \partial_{-} g(x) \\
& A_{-}=h^{-1}(x) * \partial_{-} h(x)
\end{aligned}
$$


with $g(x)$ and $h(x)$ group elements, in this case of the $U(1)$ gauge group, as defined in eq.(6). Then, the fermion determinant in (107) can be identified with the Wess-Zumino-Witten action for the $U(1)$ gauge group element $g h^{-1}$. Now, such an identification implies that one has adopted a gauge invariant regularization prescription for computing the fermion determinant, as done in the precedent section. A way to see this is the following: one can evaluate separately the left-handed and right-handed part of the fermion determinant just by working in the appropriate light-cone gauge,

$$
\begin{aligned}
& \log \operatorname{det}\left(\partial_{+}+i A_{+}\right)=W[g] \\
& \log \operatorname{det}\left(\partial_{-}+i A_{-}\right)=W\left[h^{-1}\right]
\end{aligned}
$$

Then, one can combine the two results to get the determinant of the complete Dirac operator. But in doing that, one has to add a term of the form $\int d^{2} x A_{+} A_{-}$with an a priori undetermined coefficient $\bar{a}$ since such a term is the one involved in the derivation of a finite answer from the regularized determinants. One then has

$$
\log \operatorname{det}\left(\gamma^{\mu} i D_{\mu}^{\bar{f}}[A]\right)=W[g]+W\left[h^{-1}\right]+\frac{\bar{a}}{4 \pi} \int d^{2} x g^{-1} * \partial_{+} g * h^{-1} * \partial_{-} h
$$

In the case of a gauge theory, gauge invariance fixes the arbitrary parameter $\bar{a}=1$ [17]. In the present case, since $A_{\mu}$ is not a gauge field but an auxiliary field, $\bar{a}$ remains in principle undetermined, this leading, as we shall see, to the existence of a family of solutions, a well known feature already encountered in the ordinary Thirring model.

Let us note at this point that a Polyakov-Wiegmann identity can be seen to hold in noncommutative space,

$$
W\left[g * h^{-1}\right]=W[g]+W\left[h^{-1}\right]+\frac{1}{4 \pi} \int d^{2} x g^{-1} * \partial_{+} g * h^{-1} * \partial_{-} h
$$

Using this identity, eq.(110) can be rewritten compactly as

$$
\log \operatorname{det}\left(\gamma^{\mu} i D_{\mu}^{\bar{f}}[A]\right)=W\left[g * h^{-1}\right]+\frac{a}{4 \pi} \int d^{2} x g^{-1} * \partial_{+} g * h^{-1} * \partial_{-} h
$$

where we have redefined the arbitrary parameter so that $a=\bar{a}-1$.

Concerning the integration variables, we want to write $Z$ as an integral over $g$ and $h$ and for this we have to take into account the Jacobians arising when $(108)$ is taken as a change of the path-integral variables,

$$
D A_{+} D A_{-}=J\left[g, h^{-1}\right] D g D h
$$


One can easily see that Jacobian $J\left[g, h^{-1}\right]$ coincides with the determinant of a Dirac operator in the adjoint representation. Indeed, writing for $g(\alpha)=$ $\exp _{*}(i \alpha)$

$$
g(\alpha+\delta \alpha)=g(\alpha) * \exp _{*}(i \delta \alpha)
$$

an analogous expression for $h$ one has

$$
J\left[g, h^{-1}\right] \equiv \operatorname{det} \frac{\delta A_{+}}{\delta \alpha} \operatorname{det} \frac{\delta A_{-}}{\delta \alpha^{\prime}}
$$

or

$$
J^{a d}\left[g, h^{-1}\right]=N \operatorname{det}\left(\gamma_{\mu}\left(\partial_{\mu}+-i\left\{A_{\mu},\right\}\right)\right.
$$

In passing from (115) to (116) we have again assumed that the product of left-handed and right-handed determinants can be written in terms of the determinant of the complete Dirac operator in the adjoint at the cost of taking into account an in principle undetermined, regularization dependent coefficient (here included in $N$ ).

At this point we can use our previous result on the connection between the determinant in the fundamental and in the adjoint to write

$$
\log J^{a d}\left[g, h^{-1}\right]=2\left(W\left[g * h^{-1}\right]+\frac{a}{4 \pi} \int d^{2} x g^{-1} * \partial_{+} g * h^{-1} * \partial_{-} h\right)
$$

where, for consistence, the same regularization dependent coefficient $a$ has been used.

Then, using eqs.(112), (117) we can write for $Z$

$$
Z=\int D g D h \exp \left(3 W\left[g * h^{-1}\right]-\frac{1}{2}\left(\frac{1}{g^{2}}-\frac{3 a}{2 \pi}\right) \int d^{2} x g^{-1} * \partial_{+} g * h^{-1} * \partial_{-} h\right)
$$

Now, the second term in the argument of the exponential can be made to vanish if one choses $a$ such that

$$
\frac{1}{g^{2}}-\frac{3 a}{2 \pi}=0
$$

In that case, trading $g(x)$ for a new variable $u(x)$ such that $g(x)=u(x) h(x)$, one gets

$$
Z=\mathcal{N} \int D u \exp (3 W[u])
$$


(Here, the trivial $h$ integration has been included in a normalization $\mathcal{N}$ ).

We can see this phenomenon from another point of view: for any choice of the regularization parameter $a$ there exists a particular value of the coupling constant, which we call $g^{*}$,

$$
g^{* 2}=\frac{2 \pi}{3 a}
$$

such that the Thirring model reduces to a Wess-Zumino-Witten model with "level" $k=-3$.

Now, identity (111) can be used to write, instead of (120)

$$
\begin{aligned}
Z=\int D g D h & \exp \left(3 W[g]+3 W\left[h^{-1}\right]\right. \\
& \left.-\frac{1}{2}\left(\frac{1}{g^{2}}-(3 a+1) \frac{1}{2 \pi}\right) \int d^{2} x g^{-1} * \partial_{+} g * h^{-1} * \partial_{-} h\right)(1
\end{aligned}
$$

this meaning that there exist a second critical point $g^{* *}$ such that

$$
g^{* * 2}=\frac{2 \pi}{1+3 a}
$$

at which $Z$ reduces to the partition function of two (factorized) Wess-ZuminoWitten models,

$$
Z=\int D g \exp (3 W[g]) \int D h \exp \left(3 W\left[h^{-1}\right]\right)
$$

\section{Discussion}

From the calculation of the Dirac operator determinant in a gauge field background, we have established the connection between the action for $U(N)$ fermions and the WZW action in noncommutative two-dimensional space. The bosonization recipe for fermion currents is just the trivial extension of the standard one with $*$ products replacing ordinary ones so that correlation functions of currents in the standard and the $\theta$-deformed theory will be identical. Being the WZW actions the generating functionals of fermionic current correlation functions, both actions (standard and non-commutative) are then equivalent. Moreover, a Seiberg-Witten like mapping can be constructed so that in both cases the WZW action is formally the same with the 
appropriate product of fields in each case. All these results stem from one of our fundamental formulæ(eq.(98)),

$$
\frac{d \delta W[\hat{g}]}{d \theta}=0
$$

This formula shows the independence of the WZW action on the deformation parameter $\theta$. From this, one can infer that the existence of a mass scale $\left(\left[\theta^{1 / 2}\right]=m\right)$ should not affect the scale invariance of the deformed WZW action. This is consistent with the result of [12] where it was found that the one loop beta function for a nonlinear sigma model with a Wess-Zumino term in noncommutative space vanishes at the same point as the ordinary model. In our context, this manifests through the fact that the noncommutative WZW model, when derived from the fermion determinant, gives an action at its fixed point. This behavior, which is rather evident at the fermionic level (being quadratic, the noncommutative free fermion action coincides with the standard one), is a non-trivial result at the WZW bosonic level.

On the fermion side, an interesting result is obtained when one considers the Dirac operator $\not D$ in different representations with respect to the $\theta$ deformation. We have found a factor of 2 relating the results for log det $\not D$ in the adjoint and the fundamental, reminiscent of the factor $C(G)$ that one obtains when one compares the determinant for commutative two-dimensional fermions in an $U(N)$ gauge field background, for the fundamental and the adjoint representation of $U(N)$. 
Acknowledgements: E.Moreno is partially supported by Fundación Antorchas,. This work is partially supported through grants by CONICET (PIP 4330/96) and ANPCYT (PICT 97/2285).

\section{References}

[1] A.Connes, M.R. Douglas and A.S. Schwarz, JHEP 02 (1998) 003.

[2] M.R. Douglas and C. Hull, JHEP 02 (1998) 008.

[3] N. Seiberg and E. Witten, JHEP 09 (1999) 032.

[4] S.Minwalla, M.V Raamsdonk and N. Seiberg, hep-th/9912072.

[5] M.V. Raamsdonk and N. Seiberg, JHEP 0003 (2000) 035.

[6] M. Hayakawa, Phys.Lett. B478 (2000) 394; hep-th/9912167.

[7] A. Matusis, L. Susskind and N.Toumbas, hep-th/0002075.

[8] N. Seiberg, L. Susskind and N. Toumbas, JHEP 0006(2000) 044.

[9] E. Moreno and F.A. Schaposnik, JHEP 0003 (2000) 032.

[10] C.S. Chu, Nucl.Phys. B580 (2000) 352.

[11] L. Dabrowski, T. Krajewski and G. Landi, hep-th/00003099.

[12] K. Furuta and T. Inami, hep-th/0004024.

[13] C. Núñez, K. Olsen and R. Schiappa, JHEP 0007 (2000) 030.

[14] J.M. García-Bondía and C.P. Martín, Phys.Lett. B479 (2000) 321.

[15] K. Fujikawa, Phys. Rev. Lett 42 (1979) 1195; Phys. Rev. D21 (1980) 2848.

[16] F. Ardalan and N. Sadooghi, hep-th/0002143

[17] A.M. Polyakov and P.W. Wiegman, Phys. Lett. B131 (1983) 131; ibid B141 (1984) 223. 
[18] E. Fradkin, C.M. Naon and F.A. Schaposnik, Phys. Rev. D36 (1988) 3809

[19] C.P. Martín and D. Sánchez-Ruiz, Phys.Rev.Lett. 83 (1999) 476.

[20] T. Krajewski and R. Wulkenhaar, Int.J.Mod.Phys. A15 (2000) 1011.

[21] G.Arcioni and M.A. Vázquez-Mozo, JEHP 01 (2000) 028.

[22] J.C. Le Guillou, E. Moreno, C. Núñez and F.A. Schaposnik, Nucl. Phys. B484 (1997) 484. 